\title{
FUZZIFIED PSO FOR MULTIOBJECTIVE ECONOMIC LOAD DISPATCH PROBLEM
}

\author{
N.Ramyasri ${ }^{1}$, G. Srinivasulu Reddy ${ }^{2}$ \\ ${ }^{1}$ Assistant Professor, ${ }^{2}$ Associate Professor, Dept. of EEE, Narayana Engineering College, Nellore, AP - 524004 \\ n.ramyasri10@gmail.com,gsmeghana@gmail.com
}

\begin{abstract}
Power system engineers are always striving hard to run the system with effective utilization of real and reactive powers generated by the generating plants. Reactive power is used to provide better voltage profile as well as to reduce system losses. Membership functions are written for fuel cost, losses, stability index and emission release. As minimization of real power loss over the transmission lines, an attempt is made in this paper to optimize each objective individually using Fuzzy logic approach. In this paper basic assumption is Decision Maker (DM) has imprecise or fuzzy goals of satisfying each of the objectives, the multi objective problem is thus formulated as a fuzzy satisfaction maximization problem which is basically a min-max problem. The multi objective problem is handled using the fuzzy decision satisfaction maximization technique which is an efficient technique to obtain trade off solution in multi objective problems. The developed algorithm for Optimization of each objective is tested on IEEE 30 bus system. Simulation results of IEEE 30 bus network are presented to show the effectiveness of the proposed method.
\end{abstract}

Keywords: Real power, Reactive power, losses, membership functions, fuzzy logic and trade off solution

\section{INTRODUCTION}

The real power optimization sub-problem minimizes fuel cost by controlling controllable generator outputs while keeping the PV bus voltages unchanged. The system losses, stability index and emission computed at this power dispatch are very high compared with the results obtained when respective ones are taken as objective. Similarly the reactive power subproblem deals with minimization of total transmission loss of the system by controlling all the reactive power sources such as taps, shunts etc. Thus when loss minimization is taken as objective total system losses reduces but cost, emission and stability indices are high. The emission dispatch sub problem minimizes total emission output from the fossil fuel plants by controlling the generator outputs. At this power output of generator, the cost, total system losses and stability index are high. Similarly Stability index sub problem minimizes the index by controlling the PV bus voltages and thus improves the system stability limit. But the cost, emission and system losses are very high. Thus results of all the four sub problems are conflicting with one other. This can be inferred from previous chapters. In order to meet all the four objectives, we need a compromised solution which minimizes fuel cost, emission release, total transmission and losses and improved stability limit. This trade-off solution is obtained using a fuzzy decision satisfaction maximization method.

In this paper the data of four problems are fuzzified using fuzzy Min-Max approach and then Particle swarm optimization is used to determine the final trade off solution from all these Fuzzified values. This method is tested on IEEE 30 bus system and the results are presented.

\section{PROBLEM FORMULATION}

Each particle consists of power generations of all units excluding slack bus voltages, taps and shunts encoded in it. The size of each particle is equal to sum of active power generations, no of voltages excluding slack bus, number of voltage, taps, and shunts.

Assuming the decision maker (DM) has imprecise or fuzzy goals of satisfying each of the objectives, the multi objective problem can be formulated as a fuzzy satisfaction maximization problem which is basically a min-max problem. Our task over here is to determine the compromise solution for all the four optimization sub problems. Our goal is to minimize $G(X)=$ compromised solution of $\left\{G_{1}\left(X_{1}\right), G 2\left(X_{2}\right)\right.$, $\left.\mathrm{G}_{3}\left(\mathrm{X}_{3}\right), \mathrm{G} 4\left(\mathrm{X}_{4}\right)\right\}$

While satisfying the set of constraints $\mathrm{AX}<\mathrm{B}$.

Where $G_{1}(X)$ is Fuel cost minimization sub problem.

$\mathrm{G}_{2}(\mathrm{X})$ is Loss minimization sub problem.

$\mathrm{G}_{3}(\mathrm{X})$ is emission minimization sub problem.

$\mathrm{G}_{4}(\mathrm{X})$ is index minimization sub problem.

Let $F_{1}\left(X_{i}\right)$ be the fuel cost in $\$ / h r$ for $\mathrm{i}^{\text {th }}$ control vector. $\mathrm{F}_{2}\left(\mathrm{X}_{\mathrm{i}}\right)$ be the losses in P.U for $i^{\text {th }}$ control vector $\mathrm{F}_{3}\left(\mathrm{X}_{\mathrm{i}}\right)$ be the Stability index for $\mathrm{i}^{\text {th }}$ control vector $\mathrm{F}_{4}\left(\mathrm{X}_{\mathrm{i}}\right)$ be the Emission release in $\mathrm{kg} / \mathrm{hr}$ for $\mathrm{i}^{\text {th }}$ control vector 
Let the individual optimal control vectors for the sub problems be $\mathrm{X}_{1} *, \mathrm{X}_{2}^{*}, \mathrm{X}_{3}^{*}, \mathrm{X}_{4}^{*}$ respectively. We have to find out a global optimal control vector $X *$ such that

$$
\begin{aligned}
& \left.\mathrm{F}_{1}\right|_{\mathrm{x}_{1}} \leq\left.\mathrm{F}_{1}\right|_{\mathrm{X}^{*}} \leq\left.\mathrm{F}_{1}\right|_{\left(\mathrm{X}_{2}{ }^{*}, \mathrm{x}_{3}{ }^{*}, \mathrm{x}_{4}{ }^{*}\right)} \\
& \left.\mathrm{F}_{2}\right|_{\mathrm{x}_{2}{ }^{*}} \leq\left.\mathrm{F}_{2}\right|_{\mathrm{X}^{*}} \leq\left.\mathrm{F}_{3}\right|_{\left(\mathrm{X}_{1}{ }^{*}, \mathrm{x}_{3}{ }^{*}, \mathrm{x}_{4}{ }^{*}\right)} \\
& \left.\mathrm{F}_{3}\right|_{\mathrm{x}_{3}{ }^{*}} \leq\left.\mathrm{F}_{3}\right|_{\mathrm{X}^{*}} \leq\left.\mathrm{F}_{3}\right|_{\left(\mathrm{x}_{1}{ }^{*}, \mathrm{x}_{2}{ }^{*}, \mathrm{x}_{4}{ }^{*}\right)} \\
& \left.\mathrm{F}_{4}\right|_{\mathrm{x}_{4}{ }^{*}} \leq\left.\mathrm{F}_{4}\right|_{\mathrm{x}^{*}} \leq\left.\mathrm{F}_{4}\right|_{\left(\mathrm{x}_{1}{ }^{*}, \mathrm{x}_{2}{ }^{*}, \mathrm{x}_{3}{ }^{*}\right)}
\end{aligned}
$$

The imprecise or fuzzy goal of the DM for each of the objective functions is quantified by defining their corresponding membership functions $\mu_{i}$ as a strictly monotonically decreasing function with respect to the objective function $f$ where $\mathrm{i}=1$ to 4 . In case of a minimization problem,

$\mu_{\mathrm{i}}=0$ or tends to zero, if $\mathrm{f}_{\mathrm{i}}>\mathrm{f}_{\mathrm{i}}^{\max }$ and

$\mu_{\mathrm{i}}=1$ or tends to 1 ,

if $\mathrm{f}_{\mathrm{i}}<\mathrm{f}_{\mathrm{i}}^{\text {min }}$

Where $\mathrm{f}_{\mathrm{i}}^{\max }$ and $\mathrm{f}_{\mathrm{i}}^{\mathrm{min}}$ are the unacceptable and desirable level for respectively. In our proposed approach we have considered a simple linear membership function for $f_{i}$ because none of the objectives have very strict limits. The membership function, $\mu_{i}$ for $\mathrm{i}^{\text {th }}$ objective is depicted in Fig. 1.1

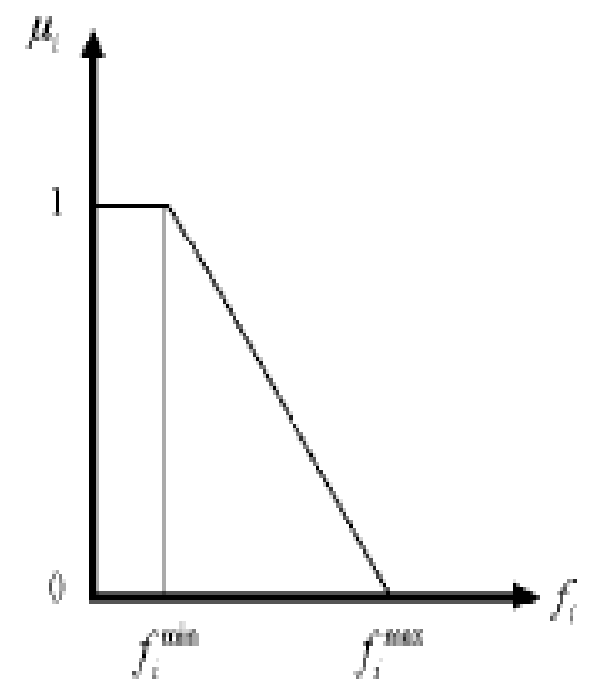

Figure 1.1 Membership function for $i^{\text {th }}$ objective
The membership function can be defined as

$$
\begin{array}{cl}
0 & \mathrm{~F}_{\mathrm{i}}>\mathrm{F}_{\text {imax }} \\
\mu_{\mathrm{i}}=\frac{\mathrm{F}_{\mathrm{i}}^{\text {max }}-\mathrm{F}_{\mathrm{i}}}{\mathrm{F}_{\mathrm{i}}^{\text {max }}-\mathrm{F}_{\mathrm{i}}^{\mathrm{min}}} & \mathrm{F}_{\text {im in }} \leq \mathrm{F}_{\mathrm{i}} \leq \mathrm{F}_{\text {imax }} \\
1 & \mathrm{~F}_{\mathrm{i}}<\mathrm{F}_{\text {imin }}
\end{array}
$$

Using Eq. (3) the membership functions can be formulated as

\subsection{Membership Function for Fuel Cost}

$$
\begin{array}{ll}
\mu_{1}=\frac{\mathrm{F}_{1}^{\text {max }}-\mathrm{F}_{1}(\mathrm{X})}{\mathrm{F}_{1}^{\text {max }}-\mathrm{F}_{1}^{\text {min }}} & \mathrm{F}_{1}>\mathrm{F}_{1 \text { max }} \leq \mathrm{F}_{1} \leq \mathrm{F}_{1 \mathrm{max}} \\
1 & \mathrm{~F}_{1}<\mathrm{F}_{1 \text { min }}
\end{array}
$$

Where $\mathrm{F}_{1 \max }=\max \left\{\mathrm{F} 1\left(\mathrm{X}_{1}{ }^{*}\right), \mathrm{F} 1\left(\mathrm{X}_{2}{ }^{*}\right), \mathrm{F}_{1}\left(\mathrm{X}_{3}{ }^{*}\right), \mathrm{F}_{1}\left(\mathrm{X}_{4}{ }^{*}\right)\right\}$ $\mathrm{F}_{1 \text { min }}=\min \left\{\mathrm{F} 1\left(\mathrm{X}_{1}{ }^{*}\right), \mathrm{F} 1\left(\mathrm{X}_{2}{ }^{*}\right), \mathrm{F}_{1}\left(\mathrm{X}_{3}{ }^{*}\right), \mathrm{F}_{1}\left(\mathrm{X}_{4}{ }^{*}\right)\right\}$

\subsection{Membership Function for Losses}

$$
\begin{array}{cl}
0 & \mathrm{~F}_{2}>\mathrm{F}_{2 \max } \\
\mu_{2}=\frac{\mathrm{F}_{2}^{\text {max }}-\mathrm{F}_{2}(\mathrm{X})}{\mathrm{F}_{2}^{{ }^{\max }-\mathrm{F}_{2}{ }^{\mathrm{min}}}} & \mathrm{F}_{2 \text { min }} \leq \mathrm{F}_{2} \leq \mathrm{F}_{2 \max } \\
1 & \mathrm{~F}_{2}<\mathrm{F}_{2 \text { min }}
\end{array}
$$

Where $\mathrm{F}_{2 \max }=\max \left\{\mathrm{F} 2\left(\mathrm{X}_{1}{ }^{*}\right), \mathrm{F} 2\left(\mathrm{X}_{2}{ }^{*}\right), \mathrm{F}_{2}\left(\mathrm{X}_{3}{ }^{*}\right), \mathrm{F}_{2}\left(\mathrm{X}_{4}{ }^{*}\right)\right\}$ $\mathrm{F}_{2 \min }=\min \left\{\mathrm{F} 2\left(\mathrm{X}_{1}{ }^{*}\right), \mathrm{F} 2\left(\mathrm{X}_{2}{ }^{*}\right), \mathrm{F}_{2}\left(\mathrm{X}_{3}{ }^{*}\right), \mathrm{F}_{2}\left(\mathrm{X}_{4}{ }^{*}\right)\right\}$

\subsection{Membership Function for Stability Index}

$$
\begin{array}{cl}
0 & \mathrm{~F}_{3}>\mathrm{F}_{3 \max } \\
\mu_{3}=\frac{\mathrm{F}_{3}^{\text {max }}-\mathrm{F}_{3}(\mathrm{X})}{\mathrm{F}_{3}^{\text {max }}-\mathrm{F}_{3}^{\text {min }}} & \mathrm{F}_{3 \min } \leq \mathrm{F}_{3} \leq \mathrm{F}_{3 \max } \\
1 & \mathrm{~F}_{3}<\mathrm{F}_{3 \min }
\end{array}
$$

Where $\mathrm{F}_{3 \max }=\max \left\{\mathrm{F} 3\left(\mathrm{X}_{1}{ }^{*}\right), \mathrm{F} 3\left(\mathrm{X}_{2}{ }^{*}\right), \mathrm{F}_{3}\left(\mathrm{X}_{3}{ }^{*}\right), \mathrm{F}_{3}\left(\mathrm{X}_{4}{ }^{*}\right)\right\}$ $\mathrm{F}_{3 \min }=\min \left\{\mathrm{F} 3\left(\mathrm{X}_{1}^{*}\right), \mathrm{F} 3\left(\mathrm{X}_{2}{ }^{*}\right), \mathrm{F}_{3}\left(\mathrm{X}_{3}{ }^{*}\right), \mathrm{F}_{3}\left(\mathrm{X}_{4}{ }^{*}\right)\right\}$ 


\subsection{Membership Function for Emission Release}

$$
\begin{array}{cl}
\mu_{4}=\frac{\mathrm{F}_{4}{ }^{\mathrm{max}}-\mathrm{F}_{4}(\mathrm{X})}{\mathrm{F}_{4}{ }^{\mathrm{max}}-\mathrm{F}_{4}{ }^{\mathrm{min}}} & \mathrm{F}_{4 \min }>\mathrm{F}_{4 \max } \leq \mathrm{F}_{4 \max } \\
1 & \mathrm{~F}_{4}<\mathrm{F}_{4 \min }
\end{array}
$$

Where $\mathrm{F}_{4 \max }=\max \left\{\mathrm{F} 4\left(\mathrm{X}_{1}^{*}\right), \mathrm{F} 4\left(\mathrm{X}_{2}{ }^{*}\right), \mathrm{F}_{4}\left(\mathrm{X}_{3}{ }^{*}\right), \mathrm{F}_{4}\left(\mathrm{X}_{4}{ }^{*}\right)\right\}$

$$
\mathrm{F}_{4 \min }=\min \left\{\mathrm{F} 4\left(\mathrm{X}_{1}{ }^{*}\right), \mathrm{F} 4\left(\mathrm{X}_{2}{ }^{*}\right), \mathrm{F}_{4}\left(\mathrm{X}_{3}{ }^{*}\right), \mathrm{F}_{4}\left(\mathrm{X}_{4}{ }^{*}\right)\right\}
$$

The maximum degree of overall satisfaction can be achieved by maximizing a scalar $\lambda$, which is the intersection of the four fuzzy membership functions.

Therefore objective function is maximization of $\lambda=\max \left(\mu_{1}\right.$, $\left.\mu_{2}, \mu_{3}, \mu_{4}\right)$.

Where $\lambda$ varies from 0 to 1 .

\section{PROPOSED ALGORITHM}

The proposed solution strategy for the multi objective problem is shown in the following algorithm

1. Reading the system data.

2. Reading the values of fixed cost, loss, index, emission for each sub-problem.

3. Forming Ybus matrix and FLG matrix for Lindex calculation.

4. Forming B1 sub matrix. Decompose B1 by Cholesky decomposition.

5. Randomly population and velocities are initialized.

6. Set Pbest $=0$ and itercount $=1$.

7. Set particle count $=1$

8. Decoding the particle, then the Decoded particle gives the values of power generations, voltage, magnitudes, tap values and shunts.
9. Forming the Ybus and B2 sub matrix. Decompose B2 by Cholesky decomposition

10. Run FDC load flow and compute loss.

11. Compute emission cost, fixed cost, and index values.

12. Fuzzifying the fuel cost, loss, emission and index obtained in step (11) using equations from Eq. 4 to 7.

13. Calculating the evaluation value of each individual in the population using Eq.(8). Compare each individual's evaluation value with its $P_{\text {best }}$. If the evaluation value of each individual is better than the previous $P_{\text {best }}$, the current value is set to be $P_{\text {best }}$.

14. Incrementing the individual count by 1 . If count < population size go to step (8).

15. The best evaluation value among the $P_{\text {bests }}$ is denoted as $g_{\text {best }}$.

16. Modifying the member velocity $V$ of each individual according to

$$
\begin{gathered}
v_{i}^{k+1}=k^{*}\left(w^{*} v_{i}^{k}+c_{1} * \text { rand }_{1} *\left(\text { pbest }_{i}-x_{i}\right)+\right. \\
\left.c_{2} * \text { rand }_{2} *\left(\text { gbest }_{i}-x_{i}\right)\right) \\
x_{i}^{k+1}=x_{i}+v_{i}^{k+1}
\end{gathered}
$$

17. Modifying the member position of each individual $\mathrm{Pi}$ according to

$$
\begin{aligned}
& \mathrm{Pi}^{(\mathrm{k}+1)}=\mathrm{Pi}^{(\mathrm{k})}+\mathrm{Vi}^{(\mathrm{k}+1)} \\
& \mathrm{Pi}^{(\mathrm{k}+1)} \text { must satisfy the constraints. }
\end{aligned}
$$

18. Incrementing iteration count by 1 .If the number of iterations reaches the maximum, then go to Step 19, Otherwise, go to Step 7

19. The individual that generates the latest $\mathrm{g}_{\text {best }}$, is the required control vector for the final trade off solution. Print the results

\section{CASE STUDIES AND RESULTS}

\section{1 IEEE 30 Bus System}

25 independent runs are made for each sub problem and the values of four factors considered at minimum value of each sub problem (data) over 25 independent runs are determined. These values for all sub problems are given in Table 1.1.

Table 1.1 results of various sub problems and final trade off solution for IEEE 30 bus system

\begin{tabular}{|c|c|c|c|c|}
\hline Optimization Problem & $\begin{array}{c}\text { Fuel Cost } \\
\mathbf{( \$ / h r )}\end{array}$ & $\begin{array}{c}\text { Losses } \\
\mathbf{M W})\end{array}$ & $\begin{array}{c}\text { Stability } \\
\text { Index }\end{array}$ & $\begin{array}{c}\text { Emission } \\
\mathbf{k g} / \mathbf{h r})\end{array}$ \\
\hline $\begin{array}{c}\text { Fuel cost minimization sub } \\
\text { problem results }\end{array}$ & $\mathbf{8 0 6 . 4 9 8 0 3 3}$ & 10.583711 & 0.272369 & 381.671279 \\
\hline $\begin{array}{c}\text { Losses minimization sub problem } \\
\text { results }\end{array}$ & 945.214704 & $\mathbf{4 . 3 2 8 3 6 7}$ & .272443 & 233.701959 \\
\hline $\begin{array}{c}\text { Stability Index minimization sub } \\
\text { problem }\end{array}$ & 897.142571 & 33.557655 & $\mathbf{0 . 1 6 2 4 4 6}$ & 375.611008 \\
\hline Emission minimization sub problem & 932.094511 & 4.404039 & 0.267070 & $\mathbf{2 2 9 . 1 4 4 8 3 4}$ \\
\hline Final trade off solution & $\mathbf{9 2 6 . 2 0 0 5 1 9}$ & $\mathbf{4 . 6 3 4 7 2 5}$ & $\mathbf{0 . 2 6 6 4 5 9}$ & $\mathbf{2 4 2 . 1 4 2 2 3 7}$ \\
\hline
\end{tabular}


System generation $=288.034725$, total load $283.4 \mathrm{MW}$. The control variables are given in table 1.2 and 1.3.and Final bus voltages, power generation, line index values are given in table1.4.

Table 1.2 Positions of tap changing transformers

\begin{tabular}{|l|l|l|}
\hline S.No & From-To buses & Tap value \\
\hline 1 & $6-9$ & 0.9 \\
\hline 2 & $6-10$ & 1.1 \\
\hline 3 & $4-12$ & 0.975 \\
\hline 4 & $28-27$ & 0.9875 \\
\hline
\end{tabular}

Table 1.3 Shunt suceptance values

\begin{tabular}{|l|l|l|}
\hline S.No & $\begin{array}{l}\text { From-To } \\
\text { buses }\end{array}$ & Shunt suceptance \\
\hline 1 & 10 & 1.009617 \\
\hline 2 & 24 & 1.0 \\
\hline
\end{tabular}

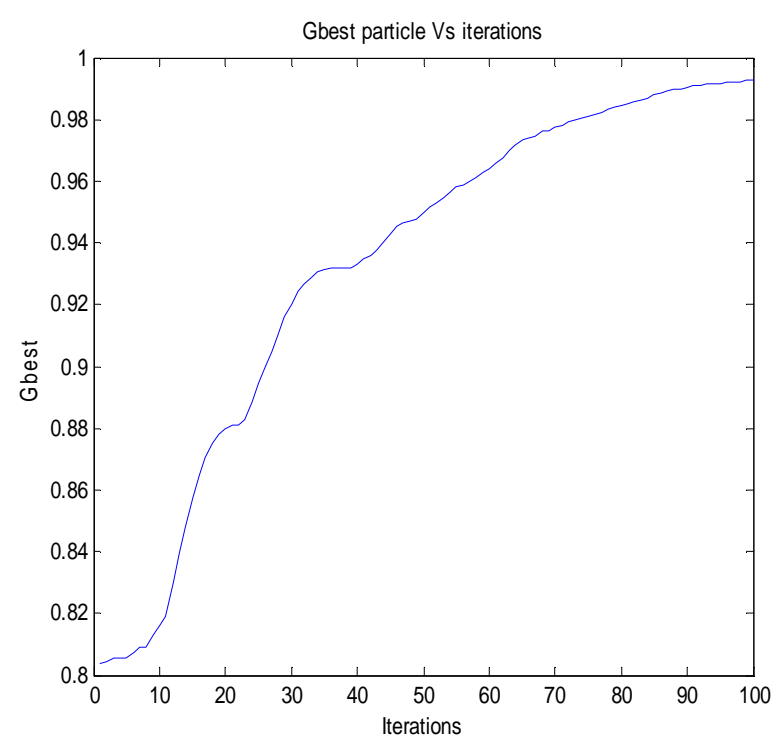

Figure 1.2 Gbest particles $(\lambda)$ Vs Iterations

Table 1.4 Final bus voltages, Power generations, Lindex

\begin{tabular}{|c|c|c|c|c|}
\hline S.No & Voltage & $\mathbf{P}_{\text {gen }}$ & $\mathbf{Q}_{\text {gen }}$ & Lindex \\
\hline 1. & 1.000000 & 0.712626 & -0.231329 & 0.000000 \\
\hline 2. & 1.003158 & 0.729346 & -0.027326 & 0.000000 \\
\hline 3. & 0.991421 & 0.500045 & 0.130286 & 0.000000 \\
\hline 4. & 0.995914 & 0.349738 & 0.633051 & 0.000000 \\
\hline 5. & 1.013388 & 0.222398 & 0.008802 & 0.000000 \\
\hline 6. & 1.000000 & 0.365994 & 0.674902 & 0.000000 \\
\hline 7. & 1.004216 & -0.000000 & -0.000000 & 0.001359 \\
\hline 8. & 0.998299 & -0.000001 & -0.000000 & 0.004968 \\
\hline 9. & 1.065043 & 0.000114 & -0.000000 & 0.091909 \\
\hline 10. & 1.042844 & 0.000594 & -0.000001 & 0.111362 \\
\hline 11. & 1.065043 & 0.000000 & 0.000000 & 0.091909 \\
\hline 12. & 1.055699 & -0.000930 & -0.000005 & 0.114096 \\
\hline 13. & 1.045549 & -0.000019 & -0.000007 & 0.122494 \\
\hline 14. & & -0.000015 & 0.000001 & 0.120544 \\
\hline
\end{tabular}




\begin{tabular}{|l|l|l|l|l|}
\hline 15. & 1.036565 & -0.000020 & 0.000002 & 0.118529 \\
\hline 16. & 1.027964 & 0.000961 & 0.000004 & 0.110391 \\
\hline 17. & 1.033049 & 0.000009 & 0.000002 & 0.113342 \\
\hline 18. & 1.025878 & -0.000003 & 0.000001 & 0.126912 \\
\hline 19. & 1.023364 & -0.000002 & -0.000000 & 0.128017 \\
\hline 20. & 1.027458 & 0.000004 & -0.000001 & 0.124354 \\
\hline 21. & 1.027112 & 0.000018 & -0.000005 & 0.115108 \\
\hline 22. & 1.026608 & -0.000500 & -0.000001 & 0.114624 \\
\hline 23. & 1.026413 & -0.000004 & -0.000000 & 0.118209 \\
\hline 24. & 1.021240 & 0.000003 & -0.000002 & 0.114837 \\
\hline 25. & 1.043109 & -0.000003 & 0.000000 & 0.099710 \\
\hline 26. & 1.025885 & -0.000002 & -0.000000 & 0.105356 \\
\hline 27. & 1.064945 & 0.000067 & -0.000003 & 0.089225 \\
\hline 28. & 0.992422 & -0.000060 & 0.000000 & 0.012565 \\
\hline 29. & 1.045961 & -0.000002 & 0.000000 & 0.104411 \\
\hline 30. & 1.034977 & -0.000008 & 0.000001 & 0.118026 \\
\hline
\end{tabular}

\section{CONCLUSIONS}

The final trade off solution is obtained. 25 independent runs are made for each sub problem and the optimal values of four objectives considered at minimum value of each sub problem over 25 independent runs are determined. In this work basic assumption made is that the decision maker (DM) has imprecise or fuzzy goals of satisfying each of the objectives, the multi objective problem is thus formulated as a fuzzy satisfaction maximization problem which is basically a minmax problem. The multi objective problem is handled using the fuzzy decision satisfaction maximization technique which is an efficient technique to obtain trade off solution in multi objective problems.

\section{REFERENCES}

[1] E. H. Chowdhury, Salfur Rahrnan, "A Review of Recent Advances in Economic IDispatch", IEEE Trans. on Power Syst., Vol. 5, No. 4, pp 1248- 1259, November 1990.

[2] Allen J. Wood, Bruce F. Wollenberg, "Power Generation, Operation, And Control", John Wiley \& Sona, Inc., New York, 2004.

[3] J. Kennedy and R. Eberhart, "Particle swarm optimization," in Proc. IEEE International Conference
Neural Networks (ICNN'95), Perth, Australia, vol. 4, pp19421948, 1995.

[4] M. R. AlRashidi, M. E. El-Hawary, "A Survey of Particle Swarm Optimization Applications in Electric Power Systems" IEEE Trans. On Evolutionary Computation Vol.13 ,No. 4,August 2009.

[5] Bo Yang and Yunping Chen, Zunlian Zhao "Survey on Applications of Particle swarm Optimization in Electric Power Systems", 2007 IEEE International Conference on Control and Automation, Guangzhou, CHINA -,pp 481-486, May 30 to June 1, 2007

[6] Zwe-Lee Gaing, "Particle Swarm Optimization to Solving the Economic Dispatch Considering the Generator Constraints", IEEE Tans. on Power Syst., Vol 18, No. 3, pp 1187- 1195, Aug. 2003.

[7] J.H.Talaq,F.El-Hawary, M.E.El-Hawary , “A Summary of Environmental/Economic dispatch algorithms" IEEE Trans. Power Syst., vol. 9, pp.1508-1516, Aug. 1994.

[8] A. Immanuel Selva Kumar, K. Dhanushkodi, J. Jaya Kumar, C. Kumar Charlie Paul, "Particle Swarm Optimization Solution to Emission and Economic Dispatch Problem", IEEE TENCON, Vol.1, pp 435-439, 2003.

[9] T.Thakur, Kanik Sem, Sumedha Saini, and Sudhanshu Sharma "A Particle SwarmOptimization Solution to NO2 and 
SO2 Emissions for Environmentally Constrained Economic Dispatch Problem", IEEE PES Transmission and Distribution Conference and Exposition Latin America, pp 1-5,2006.

[10] Papiya Dutta , A.K.Sinha- IIT Kharagpur Professor, "Environmental Economic Dispatch constrained by voltage stability using PSO” IEE ICIT 2006, IIT Kharagpur- pp. 1879-1884, 2006.

[11] Wen Zhang, Yutian Liu, "Reactive Power Optimization based on PSO in a Practical Power System" Power society engineering general meeting,vol.1, pp.239-243, 2004.

[12] Claudia Reis, F.P. Maciel Barbosa" A Comparison of Voltage Stability Indices," IEEE MELECON, Benalmádena (Málaga), Spain, pp.1007-1010, May 16-19, 2006. 